\section{REVISTA Mario Alario JURIDICA D'Filippo}

\title{
La corrupción y la moralidad pública y administrativa en Colombia
}

\author{
Corruption and public and administrative morality in Colombia
}

\author{
Alex Flórez Hernández ${ }^{1}$ iD \\ Universidad de Medellín - Colombia
}

\begin{abstract}
RESUMEN
El presente trabajo analiza la necesidad de incluir la moralidad pública y administrativa en la regulación jurídica de la actividad de los servidores públicos con el fin de establecer expectativas sociales legitimas y prevenir actos de corrupción. Esta investigación es de carácter cualitativo y su alcance es exploratorio, se realiza un análisis documental que privilegia datos oficiales al tiempo que hace uso del análisis estático de la jurisprudencia de las altas cortes. Para lograr el objetivo se realiza una presentación del alcance de la corrupción en Colombia, posteriormente se analizan los conceptos de moralidad pública y administrativa como una expectativa normativa legítima y por último se muestra que debido al enfoque sancionatorio y punitivo de la normatividad anticorrupción se ha soslayado el aporte de la moralidad pública y administrativa en la prevención de la corrupción.
\end{abstract}

Palabras clave: Corrupción, moralidad pública, moralidad administrativa, políticas públicas, Colombia.

\begin{abstract}
This paper addresses the necessity to include public and administrative morality in the legal regulation of the activity of public servants in order to establish legitimate social expectations and prevent acts of corruption. This research is qualitative in nature and its scope is exploratory: a documentary analysis is carried out that privileges official data while making use of the static analysis of the case law of the higher courts. To achieve our purpose, a presentation is made of the scope of corruption in Colombia; then, the concepts of public and administrative morality are analyzed as a legitimate normative expectation and, finally, it is shown that, due to the sanctioning and punitive approach of the anticorruption regulations, the contribution of public and administrative morality in the prevention of corruption has been ignored.
\end{abstract}

Keywords: Corruption, public morality, administrative morality, public policy, jurisprudence, legal theory, Colombia.

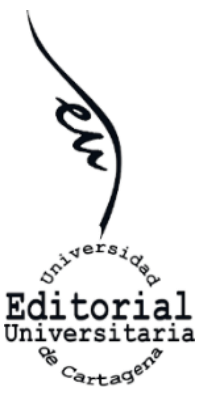

\footnotetext{
${ }^{1}$ Concejal de Medellín por el movimiento Independientes (2020-2023). Exrepresentante de los Estudiantes en la Universidad en los períodos 2014-2016 /2016-2018. Exrepresentante de los Estudiantes de Colombia ante el Consejo Nacional de Educación Superior CESU 2016. Pacifista y Cofundador del movimiento 'Jóvenes por el Sí'. alexflorezudem@gmail.com
} 


\section{INTRODUCCIÓN}

El fenómeno de la corrupción explica parcialmente el atraso de muchos países en términos de desarrollo social y económico. La corrupción pública genera desigualdad, pobreza y pérdida de credibilidad en las instituciones y órganos del Estado en la medida en que defrauda la confianza que legítimamente los ciudadanos consignan en quienes tienen a su cargo los recursos e intereses públicos y vulnera el principio de prevalencia del bien común sobre el particular. A pesar de su importancia en términos jurídicos, la corrupción no puede ser entendida de forma reduccionista como una violación estricta de los mandatos legales y constitucionales, pues esta se presenta como un fenómeno complejo que transgrede las normas legales, la moralidad, los principios y los derechos colectivos de la comunidad con la finalidad de satisfacer intereses económicos y políticos de carácter privado en detrimento del patrimonio público.

Los frágiles controles institucionales y el débil estado de derecho permiten que la corrupción se vuelva sistémica, lo que desdibuja efectivamente la separación entre los intereses públicos y privados debido a que, en algunas áreas específicas, particularmente aquellas con altos márgenes de ganancia, los grupos privados han tenido un éxito notable en la captura de los procesos de toma de decisiones políticas asegurado así que sus intereses económicos prevalezcan sobre los intereses colectivos.

Adicionalmente se ha evidenciado que, en casos extremos, todo el aparato estatal puede ser capturado y utilizado como vehículo para promover los intereses concretos de un grupo en particular (Trasparencia Internacional, 2019)

Colombia es un país que no está muy alejado de esta realidad por ello es necesario partir de una relación del alcance de este fenómeno en Colombia en los últimos años con el fin de dimensionar la problemática y su impacto en la realidad nacional. Después de realizar esta presentación, se revisará la regulación anticorrupción en el ordenamiento jurídico colombiano para resaltar la ausencia de un enfoque que acoja la moralidad pública y administrativa, por último, se analizarán los conceptos

\section{El alcance de la corrupción en Colombia}

De acuerdo con el informe anual realizado por Transparencia Internacional denominado Índice de Percepción de la Corrupción en el que se califican 180 países de acuerdo a la percepción de su nivel de corrupción en el sector público según la evaluación y análisis de expertos, empleando una escala de 0 a 100 donde 100 corresponde a corrupción inexistente y el 0 a corrupción elevada. El país, en el año 2020, ocupó el puesto 92 de 180 países con una calificación de 39 sobre 100, dos puntos por encima de la calificación 
obtenida en 2019 que fue de 37 sobre 100, ocupando entonces el puesto 96 de los 180 países evaluados.

Esta variación estadística no representa un avance significativo puesto que una calificación inferior a 50 puntos indica niveles de corrupción preocupantes en el sector público de un país y Colombia desde el año 2012 ha estado entre los 36 y los 39 puntos lo que refleja un estancamiento en la calificación realizada año tras año por esta organización. (Transparencia Internacional, 2021)

La corrupción ha dejado de ser un fenómeno coyuntural para convertirse en algo estructural y esto le ha ocasionado al país pérdidas de hasta 50 billones de pesos cada año por regalías y Sistema General de Participación, dinero que es invertido en educación, salud, y saneamiento básico de los colombianos. (Contraloría de la República de Colombia, 2018)

Entre los hechos de corrupción más sonados en Colombia en los últimos años, se encuentran el caso de Reficar que fue el mayor hallazgo fiscal del país, El Cartel de la Hemofilia, Saludcoop, Interbolsa, El Carrusel de la Contratación, El Cartel de la Toga, el caso del PAE, entre otros. A partir de lo anterior, se puede considerar que la corrupción en Colombia se ha convertido en una práctica persistente que afecta el patrimonio general de la nación y vulnera los principios de moralidad pública y administrativa.

La corrupción se ha posicionado como una de las problemáticas más relevantes del país, junto con el desempleo y la delincuencia común, con el agravante de que va en aumento con el paso del tiempo, generando serias consecuencias en la calidad de vida de los ciudadanos y en el correcto funcionamiento de las instituciones públicas, especialmente por lo que algunos autores denominan la captura del Estado. (Garay, L, 2008). Por esta razón, enfrentarse a la corrupción se ha convertido en una labor primordial toda vez que, este fenómeno trasgrede directamente los derechos fundamentales de los colombianos, el patrimonio general de la nación y debilita la democracia.

La organización Transparencia por Colombia, con apoyo de la organización a nivel internacional realiza una entrega periódica de tres informes como ejercicio de control social que tiene por objetivo distinguir los escenarios y las practicas que generan mayor riesgo de corrupción en la gestión administrativa a nivel nacional, departamental y municipal.

Estas evaluaciones se centran en tres factores de medición del índice que son: (I) Visibilidad, (II) Institucionalidad y (III) Control y Sanción, estos factores a su vez, incluyen diferentes indicadores de evaluación con base en los cuales se da la calificación final tal y como puede observarse en la gráfica 1. 


\section{GRÁFICA $1>$ FACTORES DE MEDICIÓN}
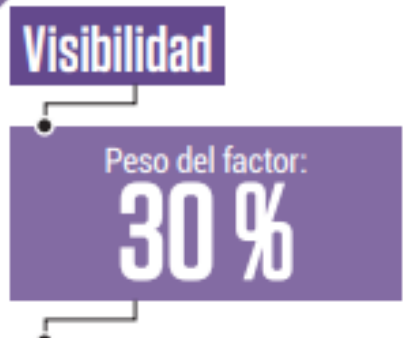

-

Evalúa riesgos asociados a la opacidad de la gestión en términos de falta de información pública, dara y oportuna. También se asocia a un bajo cumplimiento del principio de Transparencia Activa.
Institucionalidad

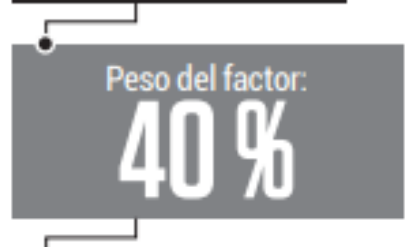

Evalúa riesgos asociados a debilidades en el cumplimiento de las normas y estándares de los procesos de la gestión administrativa, que generan un alto nivel de discrecionalidad en la toma de decisiones.
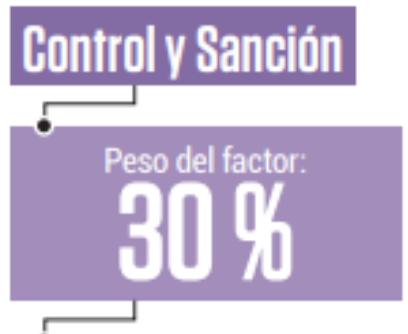

-

Evalúa riesgos asociados a la inoperancia de los mecanismos de control interno o externo de la entidad. También se asocia con las debilidades en la promoción del control social por parte de la entidad, y su relación con la ciudadanía.

Fuente: Índice de Transparencia de las Entidades Públicas 2015 - 2016. Corporación Transparencia por Colombia, 2017.

Adicionalmente, estos factores de medición están agrupados por otros indicadores a los que se les fija una calificación de 0 a 100 puntos con base en la cual se clasifica un nivel de riesgo de acuerdo al puntaje recibido finalmente como se muestra en la siguiente tabla.

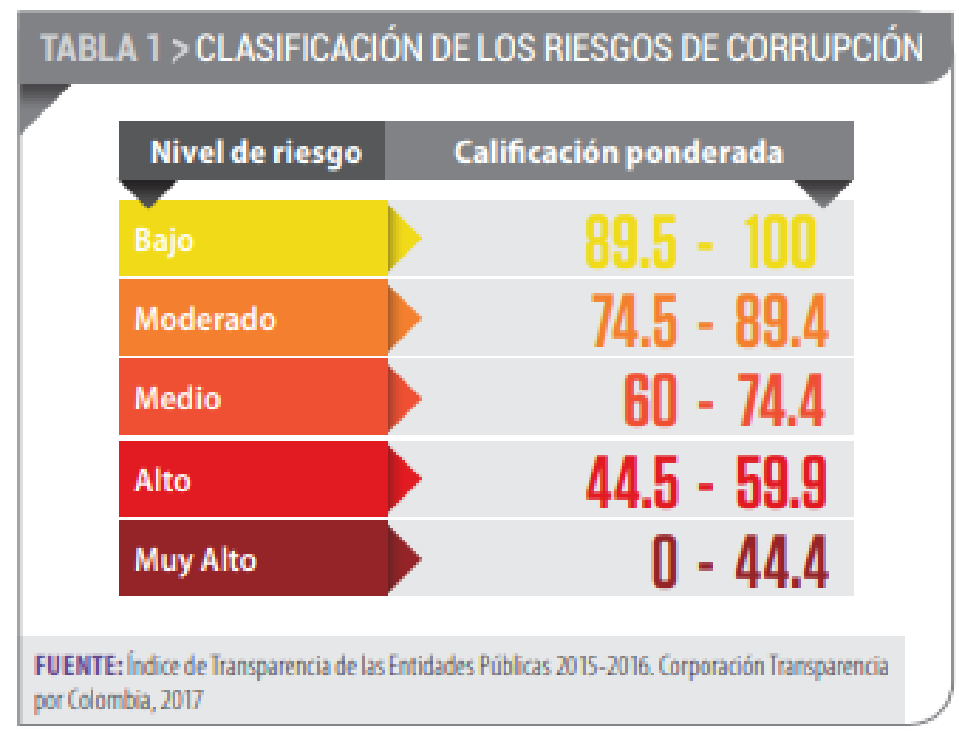

En el Índice de Transparencia Nacional 2015-2016, en el cual se evaluaron a 75 entidades del orden nacional, se estimó que el promedio general en el ranking es de $68.2 \%$, esto es, nivel de riesgo medio de corrupción; sin embargo, entidades como la Unidad de Información y Análisis Financiero (47.53\%), la Fiscalía General de la Nación (50.93\%), la Superintendencia de la Economía Solidaria (52.77\%), el Senado de la República (55.45\%), el DPS 
(Departamento Administrativo para la Prosperidad Social) (56.06), Agencia Nacional de Contratación Pública (58.84), Ministerio de Trasporte (59.41), Ministerio de Justicia (59.59\%), entre otras entidades, están en un nivel de riesgo alto de corrupción. (Transparencia por Colombia, 2017)

En cuanto al Índice de Transparencia Departamental, las 32 gobernaciones evaluadas recibieron una calificación promedio de $61.5 \%$, esto es, nivel de riesgo medio de corrupción. No obstante, cabe resaltar que, la Gobernación del Chocó (30.2\%), Gobernación de la Guajira (41.7\%) y la Gobernación de Guainía (43.0\%) se situaron en un nivel de riesgo muy alto de corrupción, mientras que diez de las gobernaciones del país se ubicaron en un nivel de riesgo alto de corrupción. Por otro lado, las contralorías departamentales recibieron una calificación promedio de $61.4 \%$ donde la Contraloría de Amazonas (34.55\%), Contraloría de Guainía (40.61\%), y la Contraloría de Vichada (43.40\%) se ubicaron en un nivel de riesgo muy alto de corrupción y otras trece en un nivel de riesgo alto. (Transparencia por Colombia, 2017). Finalmente, el Índice de Transparencia Municipal 2015-2016 arrojó datos aún más preocupantes y desalentadores toda vez que el promedio general de las 28 alcaldías en el ranking fue de $56.8 \%$, esto es, nivel de riesgo alto de corrupción. Donde ocho de las alcaldías estaban en nivel de riesgo muy alto y otras ocho en nivel de riesgo alto de corrupción. (Transparencia por Colombia, 2017)

En la publicación Grandes Hallazgos (2018), realizada por la Contraloría General, se divulgaron algunos de los casos de corrupción más controversiales en Colombia y se reveló que, en algunos de los contratos celebrados con el Estado las empresas privadas obtienen desde el $70 \%$ hasta el 100\% de las ganancias como ocurrió en el caso del Plan de Alimentación Escolar (PAE) y Cartel de la Hemofilia respectivamente. En casos como estos, son los mismos gobernantes de turno y no los contratistas los que los que se lucran de estos negocios. De acuerdo con esta publicación, durante el período 2014-2018, la Contraloría General de la República realizó 36.035 hallazgos administrativos, 13.811 con incidencia disciplinaria, 715 con incidencia penal y 4.141 con incidencia fiscal en cuantía de $\$ 21,6$ billones de pesos. (Contraloría General de la República, 2018)

Adicionalmente, según la plataforma de curaduría de información, investigación y seguimiento de la corrupción: Monitor Ciudadano, en 2018 se realizó una apropiación indebida de recursos por cerca de $\$ 17,9$ billones aproximadamente en 207 hechos de corrupción de los cuales 327 fueron exhibidos por la prensa; lo que resulta alarmante es que dicha suma de dinero es equivalente a una gran parte del presupuesto que el Estado colombiano invierte al sector educativo.

Asimismo, se detectó que entre enero del 2016 y julio del 2018 los hechos de corrupción más reportados fueron los de corrupción administrativa con un 
alto porcentaje del $73 \%$, corrupción privada con un $9 \%$ y corrupción judicial con un porcentaje del $7 \%$. Del total de actores individuales involucrados que logró identificar esta plataforma de curaduría se muestra que el 39\% fueron funcionarios públicos y un 30\% funcionarios elegidos mediante voto popular como concejales y alcaldes. En este informe se muestra también que, casi un $56 \%$ del total de los casos de corrupción reportados afectaron diversos sectores económicos, entre ellos, el sector de la educación en un 16\%, Infraestructura y Transporte en un 15\%, el sector de la Salud en un 13\% y la Función Pública en un 12\%. (Transparencia por Colombia \& Monitor Ciudadano, 2019)

Estas cifras permiten dimensionar y conocer el alcance de la corrupción en Colombia y su profundo impacto en el patrimonio económico de la nación. Se pasa ahora a analizar algunos mecanismos institucionales que han sido establecidos para luchar contra la corrupción en Colombia.

\section{Políticas públicas para la lucha contra la corrupción}

Existe diversas conceptualizaciones de lo que se concibe por políticas públicas. Con el ánimo de simplificar y siguiendo a Carlos Salazar Vargas las políticas públicas pueden ser entendidas "como el conjunto de sucesivas respuestas del Estado frente a situaciones consideradas socialmente como problemáticas" (Salazar Vargas,1995 p. 30). Sin embargo, no siempre el Estado puede enfrentar todas aquellas problemáticas debido a la falta de recursos económicos, circunstancias, presiones, intereses, etc. Por tal razón, el Estado hace frente principalmente a aquellas problemáticas que socialmente tienen más incidencia (Salazar Vargas,1995)

En Colombia, se han buscado diferentes estrategias para contrarrestar el fenómeno de la corrupción. Diversos organismos y actores han debatido por largos años sobre la idoneidad de estrategias y acciones políticas para reducir la corrupción y cómo luchar contra este fenómeno.

Un hito relevante para el estudio de la lucha contra la corrupción se da en el año 1998 con la expedición del decreto 2405 de 1998, mediante el cual se creó el Programa Presidencial de Lucha contra la Corrupción también conocido como PPLCC se planteó, entre otras, las siguientes funciones:

(I) identificar las principales causas de corrupción o ineficiencia administrativa (II) elaboración de instrumentos idóneos para constituir políticas en materia de ética y eficiencia administrativa, (III) sugerir correctivos frente a casos contra la ética administrativa (IV) recepcionar y efectuar el seguimiento de denuncias presentadas en contra de los funcionarios públicos (V) ejecutar labores de veeduría en las entidades públicas del orden nacional, entre otras. (Decreto 2405 de 1998). 
A pesar de las diversos instrumentos establecidos y efectuados por parte de este programa durante sus 10 años de vigencia, estas no tuvieron obligatoriedad ni permanencia. Ya para el año 2004 el PPLCC, presentó una iniciativa de política pública para hacer frente a la corrupción la cual tuvo por objetivo la implementación de herramientas para la prevención, investigación y sanción de actos de corrupción pública en el país partiendo de su concepción de Estado Social de Derecho. Sin embargo, esta iniciativa no se formalizó y por tanto no se logró la implementación de las acciones propuestas.

Más adelante, en el año 2011, se crea el Estatuto Anticorrupción mediante la Ley 1474 de 2011, en la cual se establecen medidas administrativas preventivas, medidas anticorrupción dirigidas específicamente al sector de la salud por ser uno de los sectores económicos más afectados por el fenómeno de la corrupción. Asimismo, establecen medidas penales en contra la corrupción pública y privada, incorpora nuevas medidas disciplinarias en contra de los funcionarios públicos y amplia los términos en que estos pueden ser investigados, adicionalmente, se definen mecanismos para combatir la corrupción en el ámbito de la contratación pública e incorpora nuevas funciones y mecanismos de intervención a los organismos especiales como la Comisión para la Moralización y la Comisión Nacional Ciudadana para la Lucha contra la Corrupción. (Ley 1474 de 2011)

Posteriormente, en el año 2013, la Presidencia de Republica con apoyo y soporte técnico de otras instituciones nacionales e internacionales, lideró la Política Pública Integral Anticorrupción la cual nace con el artículo 73 del Estatuto Anticorrupción. Esta política pública, se traza como objetivo principal "fortalecer las herramientas y mecanismos para la prevención, investigación y sanción en materia de lucha contra la corrupción en los ámbitos público y privado, con incidencia nacional y territorial, en el marco del componente nacional de la Política Pública Integral Anticorrupción (PPIA), con el fin de reducir los efectos negativos de tipo económico, social, político, jurídico y ético que se derivan de ésta, buscando impactar positivamente el desarrollo humano de los colombianos". (Documento Compes 167, 2013)

Internacionalmente, Colombia suscribió varios convenios en los cuales se comprometió a adecuar sus normatividad con el fin de combatir la corrupción, entre ellos están: "Convención de las Naciones Unidas de Lucha contra la Corrupción" Ley 970 de 2005, la "Convención Interamericana de Lucha contra la Corrupción" adoptada al ordenamiento jurídico mediante la Ley 412 de 1997 y posteriormente la Convención "Lucha Contra el Soborno Internacional" ratificada por medio de la Ley 1573 de 2012.

De la revisión de la normativa se puede verificar un claro énfasis punitivo como se puede ver en el articulado de la Convención Interamericana Contra la Corrupción, en particular el artículo III que contiene las medidas 
preventivas, el cual privilegia los sistemas de vigilancia y medidas punitivas para quienes en el pasado se han visto inmersos en prácticas de corrupción. A pesar de que estos instrumentos normativos suelen mencionar en sus exposiciones de motivos una fundamentación ética, es de notar que esta no es desarrollada en el interior de los mismos.

Ahora bien, la estrategia punitiva que se ha venido desarrollando en Colombia desde 1998 ha sido poco efectiva como muestran los altos índices de corrupción. Una alternativa a la aproximación punitiva es el fortalecimiento de la dimensión ética de la lucha anticorrupción; la moralidad pública y la moralidad administrativa se muestran entonces como una alternativa que permite armonizar las exigencias éticas de la lucha anticorrupción con las exigencias del ordenamiento jurídico, por ello se pasa ahora a analizar estas ideas en su desarrollo jurisprudencial.

\section{Los conceptos de moralidad pública y administrativa y su desarrollo en la jurisprudencia colombiana}

Al hablar de moralidad pública y administrativa es importante tener en cuenta que ambos conceptos tienen una aplicación diferenciada, aunque comparten la moral como principio preponderante. La moralidad pública se relaciona a todo el conglomerado social mientras que la moralidad administrativa se dirige a los servidores públicos frente a sus funciones administrativas.

Para el Consejo de Estado;

\begin{abstract}
"Cuando se aborda el tema de la moralidad administrativa, implícitamente se hace referencia a la corrupción, pues su significado conecta íntimamente, en uno de sus extremos, la idea de degradación, natural en un principio, y valorativa en un segundo término, de modo que, al menos desde esta última perspectiva, la corrupción está relacionada con el menoscabo de la integridad moral" (Consejo de Estado, Sección Tercera. Sentencia del 17 de junio de 2001).
\end{abstract}

Otorgar un alcance y definición a la moralidad administrativa no ha sido tarea fácil, sin embargo, la jurisprudencia del Consejo de Estado ha desarrollado una construcción conceptual de la moralidad administrativa a partir del "análisis de sus relaciones con la legalidad, así como con fenómenos como el de la corrupción, la mala fe, la ética, el recto manejo de bienes y recursos del Estado y la lucha contra propósitos torcidos o espurios, entre otros". (Consejo de Estado, Sección Tercera. Sentencia del 21 de febrero de 2007)

Para esta corporación, la moralidad administrativa despliega una naturaleza dual toda vez que actúa como principio rector de la función administrativa dotada de fuerza normativa vinculante a partir la Constitución Política de Colombia (art. 209) y la Ley 489 de 1998 (art. 3); y también, como derecho colectivo de rango constitucional que puede ser protegido mediante la acción 
popular (art. 88 Constitución Política; art. 4 Ley 472 de 1998). Esta naturaleza dual, ha sido definida por el Consejo de Estado así:

"Como principio, orienta la producción normativa infra-constitucional e infra-legal a la vez que se configura como precepto interpretativo de obligatoria referencia para el operador jurídico; y como derecho o interés colectivo, alcanza una connotación subjetiva, toda vez que crea expectativas en la comunidad susceptibles de ser protegidas a través de la acción popular" (Consejo de Estado. Sección Tercera. Sentencia de 21 de febrero de 2007 y Sentencia de 2 de septiembre de 2009).

Así, en cuanto principio se desdobla como un criterio normativo para la producción e interpretación del derecho y en cuanto derecho colectivo este puede ser exigido por los sujetos que perciben su vulneración ejerciendo la acción popular. Posteriormente, se ha indicado que los deberes que emanan de la moralidad son más exigentes que la legalidad estricta que emana del concepto de Estado de Derecho, en ese sentido en sentencia del 27 de marzo de 2014 el Consejo de Estado señaló:

"la moralidad impone que los deberes de la administración se ubiquen, más allá de las exigencias de la legalidad con que tradicionalmente se ha querido controlar su actividad, en el campo de los valores fundantes de la sociedad y que, en tanto tienen que ver con la diligencia, prudencia, pulcritud, honestidad, rectitud, seriedad y ponderación en lo discrecional, racionalidad de juicio, respeto y lealtad, en el manejo de lo que interesa a todos y propugnan por una estricta corrección en el obrar que habría de analizarse en cualquier caso, escapan a la posibilidad de que el legislador provea su contenido con antelación; pero que, en todo caso, se encuentran inmersos en lo público, por ser necesarios para que su manejo se encauce estrictamente por los fines que la carta fundamental atribuye al ejercicio del poder, como razón de ser de la organización política y de las garantías civiles" (Consejo de Estado. Sección Tercera. Sentencia del 27 de marzo de 2014).

En cuanto a las razones que fundamentan este principio como principio y derecho subjetivo manifestó:

La moralidad administrativa, en su extremo activo, pese a su titularidad difusa, consiste en la facultad de los ciudadanos para exigir, inclusive individualmente, que la actividad de la administración en sus distintas facetas -actuaciones, operaciones, hechos y contratos- se adecúe al cumplimiento de los fines estatales y se le imponga por la vía judicial, en cada caso concreto, la eficacia del derecho de que se trata; en tanto su aspecto pasivo tiene que ver con el cumplimiento de los deberes de corrección que obligan a la administración trascender, desde las exigencias formales, hasta el campo en el que la actuación se acompase con los valores y principios rectores de la sociedad política (Consejo de Estado. Sección Tercera. Sentencia del 27 de marzo de 2014). 
Respecto a la vulneración de la moralidad administrativa, esta corporación ha manifestado lo siguiente:

Con anterioridad se ha dicho que la moralidad integra al derecho, y que la moralidad administrativa integra a los valores, principios y normas correspondientes, razón por la cual cuando se trate de una vulneración a la moralidad administrativa como derecho colectivo debe evidenciarse en el proceso la violación de los dos contenidos, es decir, del contenido moral y del contenido jurídico de la norma, entendiéndose por la vulneración del primero, según el caso concreto, la mala fe, las irregularidades, el fraude a la ley, la corrupción, la desviación de poder, entre otras conductas que representan un desarrollo de conceptos morales, y que además están contempladas en el ordenamiento jurídico (Consejo de Estado. Sección Tercera. Sentencia del 21 de mayo de 2008).

Aunado a lo anterior, el Consejo de Estado ha manifestado la obligación que tiene el actor popular de demostrar mediante pruebas la presencia de prácticas corruptas o dolo para que pueda considerarse vulnerado el derecho colectivo a la moralidad administrativa. En este sentido, ha reiterado en su jurisprudencia que;

No basta demostrar la realidad de la conducta demandada (de acción o de omisión) porque ellas por sí solas, por lo general, no demuestran la amenaza o vulneración a los derechos colectivos... sería necesario que los actores probaran, además, la presencia de elementos de carácter subjetivo, contrarios a los fines de la administración (conductas amañadas, irregulares o corruptas que favorecen el interés particular a costa de ignorar los fines y principios de la recta administración), (Consejo de Estado. Sección Tercera. Sentencia del 3 de marzo de 2005).

Por su parte, la Corte Constitucional de Colombia, ha establecido que la moralidad administrativa no se predica exclusivamente del "fuero interno de los servidores públicos, sino que abarca toda la gama del comportamiento que la sociedad en un momento dado espera de quienes manejan los recursos de la comunidad y que no puede ser otro que el de absoluta pulcritud y honestidad" (Corte Constitucional, Sentencia C-046 de 1994.), en este sentido la norma que establece la moralidad administrativa debe entenderse como una estabilización de expectativas sociales, el sentido indicado por Niklas Luhmann (2012), es decir, que la moralidad administrativa constituye una expectativa normativa que la sociedad vincula con la actividad del servidor. Así entonces la moral administrativa va encaminada a servir de orientadora respecto al comportamiento que deben tener los funcionarios públicos en la realización de la función administrativa teniendo en cuenta un grupo de principios como la eficacia, la celeridad y la honestidad que del mismo modo guardan una estrecha relación con los fines del Estado dispuestos en el artículo $2^{\circ}$ de la Constitución Política de Colombia. 
Si bien estos son los preceptos a seguir para que se configure una moralidad administrativa óptima cuando sucede lo contrario, es decir, cuando el funcionario decide apartarse de este mandato, su conducta ya no está bajo el deber ser de la moralidad administrativa, debido a que encamina su función a beneficios propios desviando su poder del interés general al particular.

Frente a la moralidad pública la Corte Constitucional en la Sentencia T-301 de 2004, enuncia que este concepto tiene un alcance tripartito señalando lo siguiente;

Se le entiende como un elemento adicional para la constitución de los derechos de los ciudadanos, a su vez es una limitante de derechos constitucionales en la medida que el estado tiene facultad de restringir estos para armonizar proyectos de vida distintos dentro de una democracia y por último esta se compone por principios como; dignidad humana, la búsqueda de la paz, el pluralismo y la tolerancia, dichos principios se conectan con el estado social de derecho. (Corte Constitucional, Sentencia T-301 de 2004)

La Corte Constitucional, señala además que, la finalidad de este triple alcance se relaciona entre sí, ya que la moralidad pública articula el plano individual y social de las personas, en cuanto limita ciertos derechos individuales en aras de la protección de otro concepto que se relaciona como es el orden público con fundamento en que cada sociedad se impone unos valores mínimos que deben ser respetados por todos sus habitantes para mantener la organización social.

En conclusión, la moralidad pública es vista como aquella que armoniza los proyectos individuales para volverlos en cierto modo compatibles con el proyecto del otro y el respeto de los valores colectivos, así pues, en este principio están incluidos los mismos funcionarios públicos es su faceta de ciudadanos como cualquier otro; y la moralidad administrativa se dirige exclusivamente al sujeto que forma parte del Estado por medio de la prestación de sus servicios.

Ambos conceptos son de vital importancia dentro del Estado puesto que al existir como derecho colectivo, como es el caso de la moral administrativa, le delega al ciudadano una función crítica y analítica para ejercerlo contra quienes lo vulneren. En cuanto a la moralidad pública, la jurisprudencia se ha encargado de hacer la ponderación para evaluar en qué casos es vulnerada en aras de proteger el orden público y los valores sociales.

\section{Conclusiones}

A pesar de los esfuerzos que ha realizado el Estado y las entidades por combatir la corrupción, se puede concluir que, estos no han sido suficientes y no han dado los resultados esperados, pues la corrupción sigue siendo un 
fenómeno que afecta la vida social, económica y cultural de los colombianos afectando directamente la vida social en cuanto al suministro de bienes y servicios que se dejan de garantizar a los ciudadanos.

En el enfoque tradicional de la lucha anticorrupción se ha soslayado los principios constitucionales y legales que se ven expresados en los conceptos de moralidad pública y administrativa a pesar de la importancia que le ha sido otorgada por la jurisprudencia de las altas cortes. Un enfoque que incluya la moralidad pública y administrativa no implica un abandono de la estrategia punitiva sino que permite introducir toda una línea de defensa basada en el compromiso ciudadano y la defensa del estado social y democrático de derecho.

\section{BIBLIOGRAFÍA}

Contraloría General de la República (2018). Grandes hallazgos. Recuperado el día 25 de mayo de 2021, del sitio web de la Contraloría de la República: https://www.contraloria.gov.co/documents/20181/472298/Libro grandes+hallaz gos+CGR.pdf/6b2543f3-4faa-40c8-900d-5f47d08180ff

Consejo de Estado. (2001) Sala de lo Contencioso Administrativo. Sección Tercera. Sentencia del 17 de junio de 2001. Consejero Ponente: Alier Eduardo Hernández Enríquez.

Consejo de Estado. (2007). Sala de lo Contencioso Administrativo. Sección Tercera. Sentencia de 21 de febrero de 2007, Exp. 76001-23-31-000-2005-00549-01. Consejera Ponente: Alier Eduardo Hernández Enríquez.

Consejo de Estado. (2014). Sala de lo Contencioso Administrativo. Sección Tercera. Sentencia de 27 de marzo de 2014. Radicación No. 25000-23-15-000-201002404-01. Consejera Ponente: Stella Conto Díaz Del Castillo.

Consejo de Estado. (2005). Sala de lo Contencioso Administrativo. Sección tercera. Sentencia del 3 de marzo de 2005. Radicación No. 25000-23-27-000-2003-230401. Consejera Ponente: María Elina Giraldo Gómez.

Consejo de Estado. (2008). Sala de lo Contencioso Administrativo. Sección Tercera. Sentencia del 21 de mayo de 2008. Radicación No. 760012331000200501423 01. Consejero Ponente: Ramiro Saavedra Becerra.

Corte Constitucional. (1994). Sentencia C-046 de 1994. Magistrado Ponente: Eduardo Cifuentes Muñoz.

Corte Constitucional de Colombia. (2004) Sentencia T-301 de 2004.Magistrado Ponente: Eduardo Montealegre Lynett.

Correa Fernández, M. D. J. (2017). Corrupción en Colombia: el lado oscuro de un país en desarrollo. Revista Jurídica Mario Alario D’Filippo, 9(18), 55-74. https://doi.org/10.32997/2256-2796-vol.9-num.18-2017-2054 
Presidencia de la República. (3 de diciembre de 1998) Decreto 2405 de 1998.

Garay, L.J. (2008). La captura y reconfiguración del Estado en Colombia. Ed. Grupo Métodos. Bogotá, D.C.

Luhmann, N. (2012). Sociología del Derecho. Bogotá: Universidad Libre.

Salazar Vargas, C. (1995). Las políticas públicas. Ed. Pontificia Universidad Javeriana. Bogotá, D.C

Transparencia por Colombia. Monitor Ciudadano. (2019). Así se mueve la corrupción, radiografía de los hechos en Colombia 2016-2018. Recuperado el día 11 de mayo de 2021 del sitio web de Transparencia por Colombia: https://transparenciacolombia.org.co/Documentos/2019/Informe-MonitorCiudadano-Corrupcion-18.pdf

Transparencia por Colombia (2017). Índice de Transparencia Nacional Resultados 2015-2016. Recuperado el día 11 de mayo de 2021 del sitio web de Transparencia por Colombia: https://transparenciacolombia.org.co/2017/03/27/indice-detransparencia-nacional-2015-2016/

Transparencia por Colombia (2017). Índice de Transparencia Departamental Gobernaciones y Contralorías. Recuperado el día 11 de mayo de 2021 del sitio web de Transparencia por Colombia: https:/transparenciacolombia.org.co/Documentos/Publicaciones/indicetransparencia/ITD_2015-2016.pdf

Transparencia por Colombia (2017). Índice de Transparencia Municipal. Recuperado el día 11 de mayo de 2021 del sitio web de Transparencia por Colombia: https://transparenciacolombia.org.co/wp-content/uploads/ITEPmunicipal.pdf

Trasparencia Internacional. (2019). fomento de la integridad política para erradicar la corrupción: tres pasos para una política más limpia. Recuperado el día 25 de mayo de 2021, del sitio web de Transparencia Internacional:

Transparencia Internacional. (2021). Índice de percepción de la corrupción 2020. Recuperado el día 25 de mayo de 2021, del sitio web de Trasparencia Internacional: $\quad$ https://www.transparency.org/en/press/2020-corruptionperceptions-index-reveals-widespread-corruption-is-weakening-covid-19response-threatening-global-recovery 